\title{
A Novel Hope for Alopecia Totalis Patients: Case Report
}

\author{
Manal Elsayed $^{\mathrm{a}}$ Laila Al Otaibi $^{\mathrm{b}} \quad$ Nael Quraishy $^{\mathrm{a}} \quad$ Afzalhussein Yusufali $^{\mathrm{a}}$

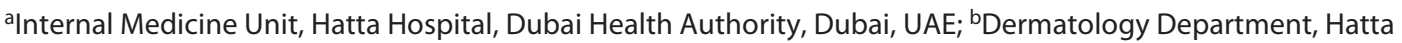 \\ Hospital, Dubai Health Authority, Dubai, UAE
}

\section{Keywords}

Janus kinase inhibitor $\cdot$ Tofacitinib $\cdot$ Alopecia totalis $\cdot$ Case report

\section{Abstract}

Alopecia areata (AA) is a common autoimmune disorder causing nonscarring patchy hair loss. Alopecia totalis (AT) is a severe variant of AA. Although there are several available treatment modalities for AA, efficacy of most of them is not satisfactory in case of AT. Recently, several case reports and series and small open-label studies have shown efficacy of oral Janus kinase (JAK) inhibitors as treatment for AT. Tofacitinib is one of the JAK inhibitors, which is an approved drug for treatment of rheumatoid and psoriatic arthritis.

In this case report, we have treated a 24-year-old girl who had juvenile chronic arthritis and developed AT. She was treated for 3 years with different modalities without satisfactorily results. We treated her with tofacitinib $5 \mathrm{mg}$ orally twice a day and assessed its efficacy and adverse effects if any. We monitored scalp hair regrowth of the patient using the score of severity of alopecia tool. The patient tolerated the treatment well; hair regrowth started from the 4th week and full regrowth attained by the 9th month of the treat- ment. No serious adverse effects were noticed. Tofacitinib can potentially be considered as an effective and well-tolerated treatment for AT; however, larger studies are needed to address its long-term efficacy.

(C) 2020 The Author(s)

Published by S. Karger AG, Basel

\section{Introduction}

Alopecia areata (AA) is an autoimmune disease that targets anagen hair follicles causing nonscarring hair loss through chronic hair follicle inflammation. It affects both male and female equally [1]. Although it presents as asymptomatic localized hair loss it can develop to full loss of scalp hair as alopecia totalis (AT) or the entire body hair loss as alopecia universalis (AU). Immune system plays a role in the pathogenesis of AA mediated by CD8+ T cells [2]

Usually, the first-line treatment for mild patchy AA is topical and intralesional steroids. Systemic steroids are used in moderate to severe AA, but they have many side effects and high relapse rate and are not effective in case of AT or AU [3].

karger@karger.com www.karger.com/dmj

Karger $\stackrel{\text { ' }}{=}$

BOPEN ACCESS
C) 2020 The Author(s).

Published by S. Karger AG, Basel

This article is licensed under the Creative Commons AttributionNonCommercial-NoDerivatives 4.0 International License (CC BYNC-ND) (http://www.karger.com/Services/OpenAccessLicense) Usage and distribution for commercial purposes as well as any distribution of modified material requires written permission.
Dubai (United Arab Emirates)

msrezzek@dha.gov.ae 
SALT score: sequential analysis

Fig. 1. Showing score of severity of alopecia tool (SALT).

\begin{tabular}{|l|l|l|l|l|l|}
\hline Date & Top: 40\% & Back: 24\% & Left side: 18\% & $\begin{array}{l}\text { Total \% of } \\
\text { hair loss }\end{array}$ \\
\hline
\end{tabular}

Janus kinase (JAK) inhibitors are drugs that inhibit the activity of one or more of the JAK family of enzymes, so interferes with the intracellular signaling pathway. Tofacitinib is a specific inhibitor of JAK3 [4]. In the last few years, JAK inhibitors have been investigated as potential treatment for AA. The rare but possible side effects of JAK inhibitors are anemia, thrombocytopenia, neutropenia, deranged lipid profile, bacterial and fungal infections, reactivation of $\mathrm{TB}$, and herpes zoster.

\section{Case Report}

The patient is a girl of 24-year old who is known to have juvenile chronic arthritis, polyarticular type since the age of 8 years. She also has Hashimoto thyroiditis. Her mother has diabetes and AU for more than 20 years. The patient had been treated with methotrexate and etanercept for 10 years. She attained remission, and her disease activity score 28 (DAS 28 score) was $<2.6$. She is off medication with complete remission and no joint symptoms since last 6 years. However, she is on thyroxine $100 \mathrm{mcg}$ as replacement therapy. She experienced scalp hair fall that deteriorated to complete loss 3 years before she was seen in our clinic. She had consulted many dermatologists and had tried different treatment modalities that including intralesional injection of steroid, minoxidil, and systemic steroid without much improvement. This patient was suffering from severe AA represented clearly as AT. She was subjected to baseline laboratory evaluation including complete blood cell count with differential, comprehensive metabolic panel, fasting lipid panel T-spot and screening for HIV, and hepatitis $\mathrm{B}$ and $\mathrm{C}$ viruses, before starting on JAK inhibitor tofacitinib $5 \mathrm{mg}$ orally twice a day. The patient has been followed up and as- sessed monthly. The percentage of patient's scalp hair loss is being determined using the score of the severity of alopecia tool (Fig. 1). The patient's scalp hair started its regrowth from the 4 th week, and the patient attained the full regrowth of her scalp hair by the 9th month of the treatment (Fig. 2). There were no adverse effects of the drug.

\section{Discussion/Conclusion}

The outcome was very successful and encouraging especially as it was an atypical therapeutic approach of AT at our hospital. The effect on our patient was visible and profound. She was depressed before the treatment and is proud, happy, and confident now.

Immune system plays a role in the pathogenesis of AA mediated by CD8+ NKG2D+ T effector memory cells through JAK signaling pathway [5]. There are several case reports and series showing the efficacy of JAK 3 inhibitor and tofacitinib in treatment of AT [5]. A retrospective study with 90 patients with AT and AU treated with tofacitinib has shown encouraging results over 4-18 months of treatment with no serious adverse events [6]. Unfortunately, recurrence rate on stopping treatment is high and the role of long-term treatment unknown. The literature is limited and does not answer many key questions. Large randomized trials are needed to confirm the efficacy, appropriate dosage, length of treatment, appropriate way of treatment application (oral and/or topical), and safety of such therapy. JAK inhibitors/tofacitinib is increasingly 
Fig. 2. Showing sequential photos before and after treatment.

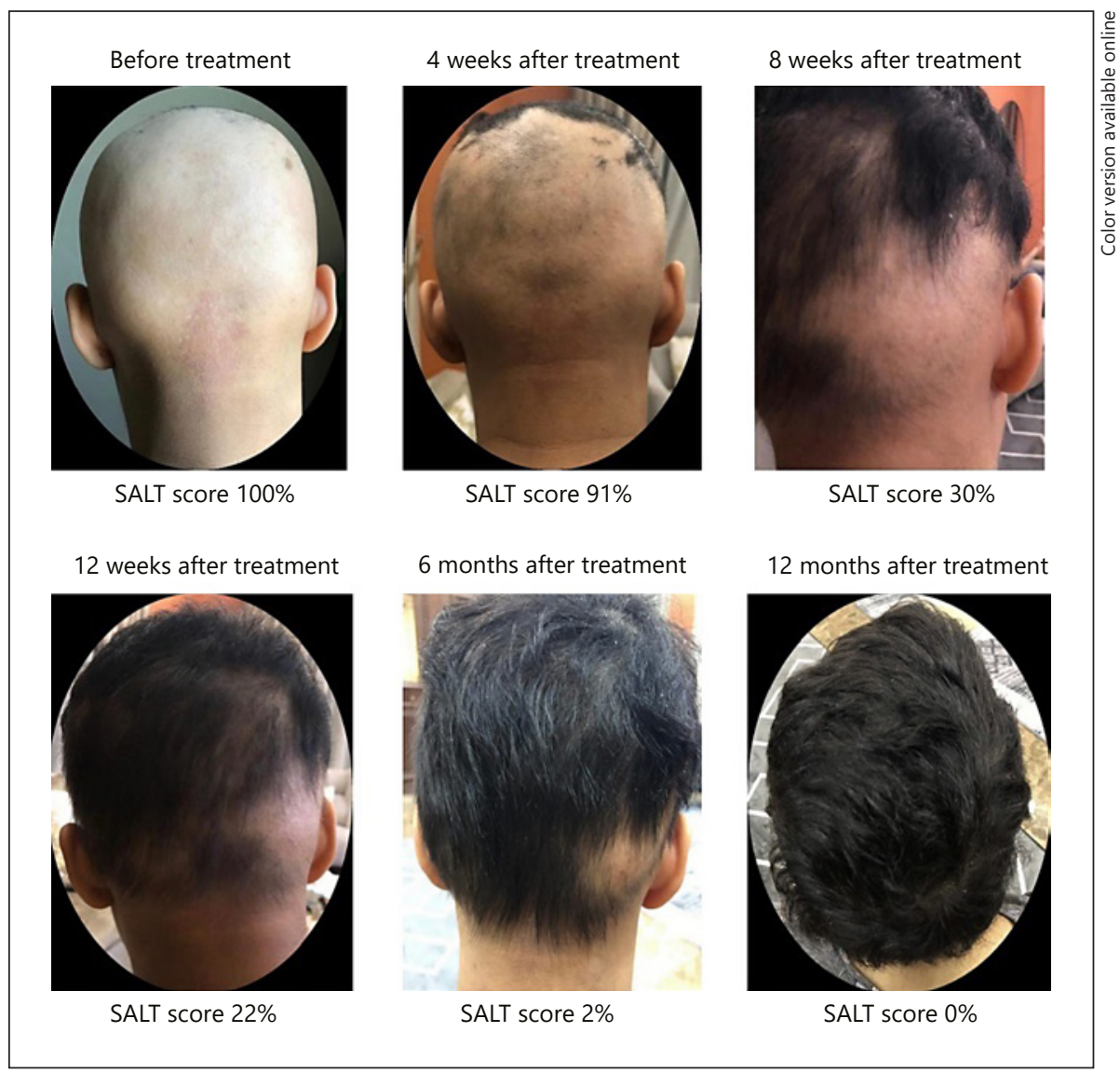

being used as new therapeutic approach to address AT disease. However, these are outside the stated indications. With formal trials and if successful as recommended in guidelines and greater awareness, more of our patients will benefit.

\section{Acknowledgement}

I would like to express my deep thanks to Dr. ASA for his help throughout writing this paper.

\section{Statement of Ethics}

Ethical conscent has been taken fom the patient to publish this case.

\section{Conflict of Interest Statement}

The authors have no conflicts of interest to disclose.

\section{Funding Sources}

The authors did not receive any funding.

\section{Author Contributions}

MSR made the diagnosis, treated, and followed up the patient. All the rest helped with the case, helped with the drafting the case report, and revising it critically. They approve the version to be published.

\section{Declaration of Patient Consent}

The patient has given written consent that her unidentified images and other related clinical information will be reported in the journal. The patient understands that her name and initial will not be published and all due efforts will be made to conceal her identity; however, anonymity cannot be fully guaranteed. 


\section{References}

1 Mirzoyev SA, Schrum AG, Davis MarkD P, Torgerson Rochelle R. Lifetime incidence risk of alopecia areata estimated at $2.1 \%$ by rochester epidemiology project, 1990-2009. J Invest Dermatol. $2014 \mathrm{Apr} ; 134(4): 1141-2$.

2 Pratt CH, King LE Jr, Messenger AG, Christiano AM, Sundberg JP. Alopecia Areata. Nat Rev Dis Primers. 2017;3:17011.
3 Jahn-Bassler K, Bauer WM, Karlhofer F, Vossen MG, Stingl G. Sequential high- and lowdose systemic corticosteroid therapy for severe childhood alopecia areata. J Dtsch Dermatol Ges. 2017;15(1):42-7.

4 Kontzias A, Laurence A, Gadina M, O'Shea JJ. Kinase inhibitors in the treatment of immune-mediated disease. F1000 Med Rep. 2012;4:5.
5 Jabbari A, Dai Z, Xing L, Cerise JE, Ramot Y, Berkun Y, et al. Reversal of alopecia areata following treatment with the JAK1/2 inhibitor baricitinib. EBioMedicine. 2015;2(4):351-5.

6 Liu LY, Craiglow BG, Dai F, King BA. Tofacitinib for the treatment of severe alopecia areata and variants: a study of 90 patients. J Am Acad Dermatol. 2017;76(1):22-8. 